\title{
Evidence-based learning in general dental practice
}

\section{Malcolm Bruce}

All patients have the right to expect a high standard of care from their dentist ${ }^{1}$ and the maintenance of these standards must involve a commitment to keep up to date. ${ }^{2}$

General dental practitioners, like myself, will soon be required to record a minimum of 35 hours personal study per year. This precious reading time must be productive, interesting and preferably straightforward. One way to do this is to relate it to knowledge needs or a clinical problem. This problem-based approach is at the heart of evidence-based dentistry. It has been described, as a life long, self-directed, problem based learning process. Table 1 depicts the stages of this which will be familiar to many readers.

$\begin{aligned} & \text { Table I Stages of the evidence based learning } \\ & \text { process }\end{aligned}$
Identify knowledge needs
Formulate a focused question
Search for evidence
Appraise the evidence
Act
Evaluate

Knowledge needs

It is important to question clinical techniques, particularly new ones, and to be aware of the effectiveness of our interventions.

Recently, a colleague in the practice asked me to order minocycline gel for treating periodontal pockets. I ordered it but then realised there was a knowledge need and that I should have determined its effectiveness first. So I considered what my question should be.

\section{Formulating a focused question}

Focused questions are easy to formulate. The important points to note are the clinical problem, category of patient, the intervention being considered, an alternative intervention for comparison and the clinical outcome being studied.
In the case of minocycline gel, my question was:

In adult patients with moderate to severe periodontitis how effective is topical minocycline gel as an adjunct to non-surgical periodontal treatment?

The constituent parts are detailed in table 2. I decided to use bleeding on deep probing as the main outcome variable to consider.

\section{Searching}

Once the question has been formulated searching for the information to answer it is made easier, more focused and usually produces a result more quickly. There are a number of ways to go about it. The ones I use are detailed below.

\section{Do-it-yourself}

Internet sites (e.g. HealthGate) which provide free access to Medline are probably the easiest and cheapest way for a GDP to carry out his or her own searches. If you don't have a PC with an internet connection cybercafes can be fun or you can visit a District hospital library where practitioners with an NHS commitment are usually able to use the facilities for searching.

Searching for evidence can be time consuming but a number of organisations provide hands-on courses in this area (CASP and CEBD and some libraries) some local librarians will also offer to help develop your searching skills.

The New National Electronic Library for Health (http://www.nelh.nhs.uk) which is currently being developed and is intended to be one of the world's great libraries will open later in the year. This library will focus on quality information and will be available to dental practitioners as well as medical practitioners and other health care providers.

Doing your own searching must be recommended as it is interesting, often rewarding and gives a first hand impression of the numbers and types of published papers available.

\section{Getting help}

To save time and effort you can ask a librarian to carry out a search for you. It is helpful to use a librarian who has experience within dentistry, the BDA library offers this service.

The Cochrane Oral Health Group in Manchester have now gathered over 6,000 randomised controlled trials and may help with searching. The School of Health and Related Research (ScHARR) in Sheffield is an excellent internet site which will guide and carry out searches for members.

\section{Systematic reviews and} meta-analyses

Any search carried out by ourselves or by a librarian on our behalf will be superficial when compared with a search carried out during a properly conducted systematic review or metaanalysis. These searches can take weeks or months and involve hand searching. If available, the retrieval of a properly conducted review or meta-analysis
Table 2 Parts of a focused question regarding minocycline gel.

Patient/problem

Intervention

Alternative intervention Non-surgical periodontal treatment with topical placebo gel

Outcome
Adult patients with moderate to severe periodontitis Topical minocycline gel as an adjunct to non-surgical peridontal treatment Absence of bleeding on deep probing 
which addresses your question is imperative as the search will be thorough and the evidence already subjected to a quality filter.

\section{Critical appraisal}

A detailed explanation of critical appraisal is beyond the scope of this short example. However, essentially you need to answer three questions: -

- Is the paper valid?

-What are the results?

- Are the results relevant to my patient(s)?

My search for information about minocycline gel revealed four randomised controlled trials. Two of these evaluated bleeding on deep probing using the papillary bleeding index, a qualitative method and therefore difficult to include in a quantitative analysis. The other two trials evaluated bleeding on deep probing by its presence or absence, a quantitative method. Unfortunately, validity was questionable for one of these trials as over $20 \%$ of its subjects were lost to follow up at the end of the study. Three papers were placed to one side. All had found no statistically significant difference between the experimental and control groups for this outcome variable.

\section{Absolute Risk Reduction}

The fourth paper I judged to be valid in respect of my question. ${ }^{3}$ There were 30 subjects of which 26 completed the trial. In the control group (non-surgical treatment plus placebo gel) $27.1 \%$ of the periodontal pockets which demonstrated bleeding on deep probing at the beginning of the trial were still demonstrating this outcome variable at the end of the trial, this is the Control Event Rate (CER). The corresponding rate in the experimental group (non-surgical treatment plus minocycline gel) was $23.3 \%$ (Experimental Event Rate, EER). CER minus EER is the absolute difference in the rates of bleeding on deep probing between control and experimental groups at the end of the trial, this is the Absolute Risk Reduction (ARR) and was $3.8 \%$, with a $95 \%$ confidence interval (CI) of $5.8 \%$ to $13.4 \%$. This is the range of values within which we can be $95 \%$ sure that the true population value lies. ${ }^{4}$
Numbers Needed to Treat (NNT)

The numbers needed to treat is calculated by taking $\frac{1}{\mathrm{ARR}}$

that is $\frac{1}{3.8 \%}$ or 26 (ref. 5).

Non-surgical periodontal treatment is effective but to achieve an outcome of one extra pocket not bleeding on deep probing, 26 periodontal pockets demonstrating this clinical variable would need to be treated with minocycline gel.

CI for NNT is obtained as reciprocal of CI for ARR, 95\% CI for this NNT gives a range from 7.4 to infinity.

\section{If available, the retrieval of a properly conducted review or meta-analysis which addresses your question is imperative as the search will be thorough and the evidence already subjected to a quality filter.}

Act

The benefits of using minocycline gel for this outcome variable are outweighed by the cost and the risks associated with topical antibiotics. Consequently I have no plans to make further purchases without more convincing evidence. A more recent search by the BDA library has retrieved no additional randomised controlled trials of this delivery system.

\section{Evaluation}

As well as any improvements in cost effectiveness from not using minocycline gel further educational gains can be made for the keener practitioner. I asked a librarian about my search strategy to see if by improving the search I could have found more papers. She confirmed that the strategy was good but I could have repeated it in databases other than Medline. In addition I contacted one of the authors about my conclusions from the trial. He agreed with the NNT but explained that in a less well controlled environment, such as a community trial, the treatment would be likely to be less effective.
Sharing knowledge about clinical effectiveness

Using this process whenever there is a knowledge need would be beneficial but is also time consuming. Secondary journals such as this supplement to the BDJ provide the best available evidence in a rapidly accessible form. Evidence-based Dentistry can be read knowing that rigour has been applied to the published literature.

The Centre for Evidence Based Medicine has a website with a 'CATbank'. This is a repository for critically appraised topics, or CATS, similar to the example above with calculations of risk reductions and numbers needed to treat.

The scope for producing CATs like these is vast. Last year two groups of Vocational Dental Practitioners were asked to identify gaps in their knowledge regarding effectiveness and they produced some forty questions, some of which are noted in table 3.

\section{Table 3 Some examples of knowledge needs identified by vocational dental practitionaers \\ How effective is Periochip? \\ How effective is Dyract? \\ Do compomers bond to amalgam restorations? \\ Which is the best composite to use? \\ Can teeth be etched for less than 20 seconds? \\ Which is the best restorative material for the deciduous dentition - amalgam or glass ionomer?}

The strength of a profession lies in the quality of scientific knowledge and in the care and rigor with which it is applied in practice. If the standing of dentistry is to be maintained and enhanced the evidence based approach must be at its core.

1 General Dental Council: Maintaining Standards 1998, 3.2.

2 General Dental Council: Maintaining Standards 1998, 1.3.

3 Graca $M$ A et al. A randomized controlled trial of a $2 \%$ minocycline gel as an adjunct to non-surgical periodontal treatment, using a design with multiple matching . criteria. J Clin Periodont 1997; 4: 249-253.

4 Sackett D L et al. Evidence Based Medicine, How to Practice and Teach EBM. p228. 1997; Churchill Livingstone: London.

5 Evidence Based Dentistry 1997; 1: 27. 